\title{
Effect of main part size and disparity distribution on visual comfort of Stereoscopic images
}

\author{
Zhibin Su, ${ }^{1,2,3, a}$, Fangju Zou ${ }^{1,2,3, b}$, and Ren Hui ${ }^{1,2,3, c}$ \\ ${ }^{1}$ School of Information and Engineering, Communication University of China \\ ${ }^{2}$ Key Laboratory of Acoustic Visual Technology and Intelligent Control System, Ministry of Culture \\ ${ }^{3}$ Beijing Key Laboratory of Modern Entertainment Technology

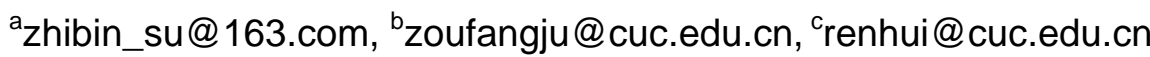

Keywords: stereoscopic images, main part size, disparity, subject assessment.

\begin{abstract}
Visual comfort is one of the important indexes to evaluate the image quality and viewing experience in the process of viewing stereoscopic video and images. This paper mainly investigated the effect of the main part size and disparity distribution type of stereoscopic images on visual comfort through two subjective evaluation experiments. The experiment results confirmed that the main part size and disparity space distribution type are two important factors which can affect visual comfort, and they also proved the influence trend of these factors on visual comfort in different disparity conditions. Our experiments have important guiding significance for the establishment and improvement of the relevant objective evaluation experimental model and acquisition and display technology of stereoscopic images.
\end{abstract}

\section{Introduction}

With the popularization of 3D film and 3D TV, the stereo video technology is improved in a rapid speed. At the same time, people's requirement of stereo video has become increasingly high. They have the higher standard and pursuit about the quality of the image and the viewing experience. Numerous studies confirmed that the visual comfort is one of the most important indexes in the measurement of image quality and the viewing experience[1]. Literature [2][3] proposed that visual comfort refers to a subjective feeling of discomfort accompanied by physiological changes, such as: eyestrain, difficulty in focusing or blurred vision, and headaches etc.

Visual comfort evaluation method is divided into two categories, objective assessment and subjective assessment method. As the reference for establishing the objective model, subjective assessment for the factors of visual comfort is studied in this paper. It is known that researchers have found many factors that will affect the visual comfort. Based on the characteristics of stereo images, We intended to investigate the influential factors from characteristics of image pairs and image itself, including to parallax amplitude, the main part size, the disparity spatial distribution and temporal parallax change etc. Among the factors of image pairs, disparity and its related characteristics have been the emphases of current research. For example, literature [4] [5] proposed that oversize disparity magnitude would cause visual discomfort. Literature [6] proved that the comfort decreases when the absolute value of disparity magnitude increases. Literature [7] confirmed that images were more comfortable to watch when the bottom of the image appeared closer and the top of the image appeared further away. For the characteristics of the image itself, literature [3] proved that the contrast ration (CR) of the main part and the background would affect the visual comfort. Literature [8] investigated the effect of the main part size on visual comfort, and proved that the comfort would improve with the size increasing when the main part size is small; however, the research had a lack of larger size effect on visual comfort. Therefore, according to the analysis of current situation, this paper mainly investigated the effect of disparity distribution types and the main part size on visual comfort through two subjective evaluation experiments. 


\section{Experiment Materials}

The experiment about main part size. Firstly, the effect of main part size on visual comfort of stereoscopic images was studied under different disparity scales. According to the literature [3], the foreground and background gray scale contrast was set to $0.5(\mathrm{CR}=0.5)$. As the main part of the image, it had three levels of disparity magnitude: 3\% (about 30 arc min), 5\% (about 5 arc min), 7\% (about 70 arc min), and two types of disparity: crossed disparity and uncrossed disparity. The size of the main part of the image was divided into eight grades. Therefore, there were 48 test sequences, and each of the eight sequences was for a group. And then the pixel of the main part was: $1(80 * 80) 、 2(260 * 260) 、 4(310 * 310) 、 6(460 * 460) 、 8(620 * 620) 、 10(760 * 760) 、 12(920 * 920)$, $14(1070 * 1070)$ 。 The experimental material is show in Fig. 1.

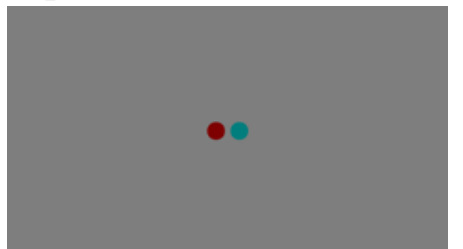

( a )

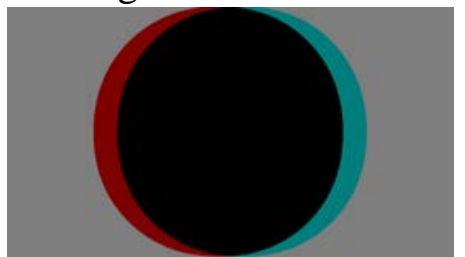

(b)

Fig. 1 The display sequence in main part size experiment ( a The main part size was $80 * 80$

b The main part size was $1070 * 1070)$

A modified version of the Single Stimulus (SS) method was used for the assessment. The order of 48 sequences was randomly arranged, and it was watched twice by every observer with different orders. There were 20 participants (10 boys and 10 girls), with an average age of 24 years. All of them were not expert and proved to have normal stereoscopic vision by passing relevant stereo-acuity and multi-stereograph Tests. Table 1 shows the 5-point scales of visual comfort which is the international standard in the experiment[3].

Table 1 Evaluation scale of visual comfort

\begin{tabular}{|c|c|c|}
\hline \multicolumn{2}{|r|}{ Description of the Scale } & score \\
\hline Very comfortable & $\begin{array}{l}\text { The viewing experience is in complete accord with } \\
\text { normal human eye; comfortable and perfect }\end{array}$ & 1 \\
\hline Comfortable & $\begin{array}{l}\text { The viewing experience is familiar with human eye, no } \\
\text { discomfort }\end{array}$ & 2 \\
\hline $\begin{array}{l}\text { Perceptibly uncomfortable } \\
\text { but acceptable }\end{array}$ & $\begin{array}{l}\text { The critical point that causes discomfort feeling. (scores } \\
\text { fall in this range should be pay attention) }\end{array}$ & 3 \\
\hline $\begin{array}{l}\text { Clearly uncomfortable } \\
\text { and unacceptable }\end{array}$ & $\begin{array}{c}\text { The critical point that causes discomfort and } \\
\text { unacceptable feeling. (scores fall in this range should be } \\
\text { avoided) }\end{array}$ & 4 \\
\hline $\begin{array}{c}\text { Extremely uncomfortable } \\
\text { and extremely } \\
\text { unacceptable } \\
\end{array}$ & & 5 \\
\hline
\end{tabular}

The final test sequences were generated by the Vegas Sony software and the format of $1920 * 1080$, side by side (half). The intention of the assessment and the negative effect could be caused by the experiment were not concealed for observers who participated in experiment. The observer can stop the assessment at any time if he felt uncomfortable[9]. They were asked to watch the arranged sequences with polarized glasses at the distance of $1.8 \mathrm{~m}$.

The experiment about disparity distribution types. This experiment mainly studied the effect of the spatial distribution of the disparity on the visual comfort. There were five range of magnitude distribution which were 20, 30, 40, 50, 60 arc min. In addition, disparity distribution types were divided into five types according to the simulation of natural images, the five distribution types were as follows: 
Type_a: Round and triangle : within the screen, uncrossed disparity

Type_b: Round: within the screen, uncrossed disparity. Triangle: on the screen, zero disparity

Type_c: Round: within the screen, uncrossed disparity. Triangle: outside the screen, crossed disparity

Type_d: Round: on the screen, zero disparity. Triangle: outside the screen, crossed disparity

Type_e: Round and triangle: outside the screen, cross disparity

Finally, the combinations of two factors generated 25 sequences, which are shown in Fig. 2

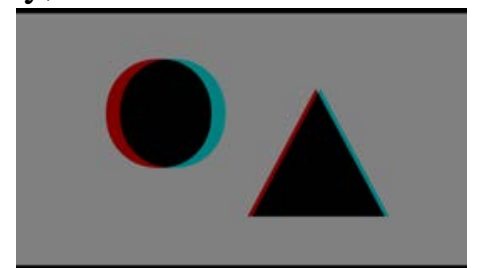

( a )

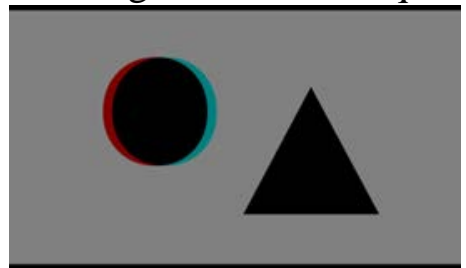

(b)

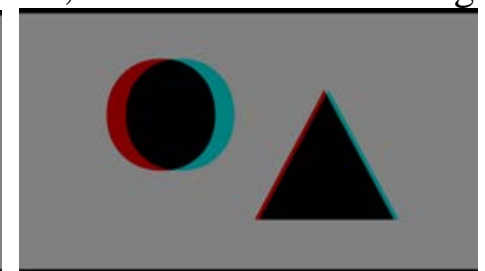

( c )

Fig. 2 the display sequence in disparity distribution experiment ( a disparity range: 20 arc min, type_a b disparity range 20 arc min, type_b c disparity range 30 arc min, type_a)

There were 20 participants (10 boys and 10 girls), with an average age of 24 years. In this experiment, the evaluation method, scoring table and requirements of the observer were the same as the experiment before.

\section{Analysis of the experimental result}

The effect of main part size on visual comfort of stereoscopic images. The rating scores should be processed according to literature [7]. Finally, there were 15 observers' rating data kept for the experiment. Through an multivariate repeated measures analysis of variance, for main part, F ( 7 , $98)=3.148, \mathrm{P}<0.01$, so the effect of main part is significant. For the factors of parallax and parallax type, $\mathrm{P}>0.05$, so the effect is not significant. Fig. 3 shows the main experimental results of the mean opinion score (MOS). It can be seen form Fig. 3 that the observers feel more comfortable with the increasing of main part size when it was small; however, when the comfort degree achieves the extreme point, it begin to decline with the size increasing. Fig. 3 (a) also indicated that the position where the observer feet most comfortable is different at different disparity magnitude. The larger the disparity is, the smaller the comfortable size of the main part is.

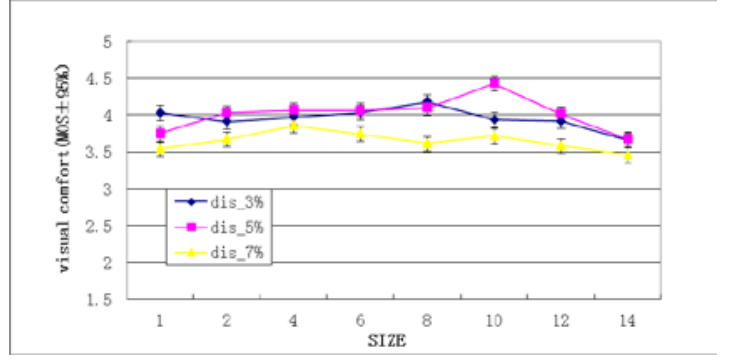

( a )

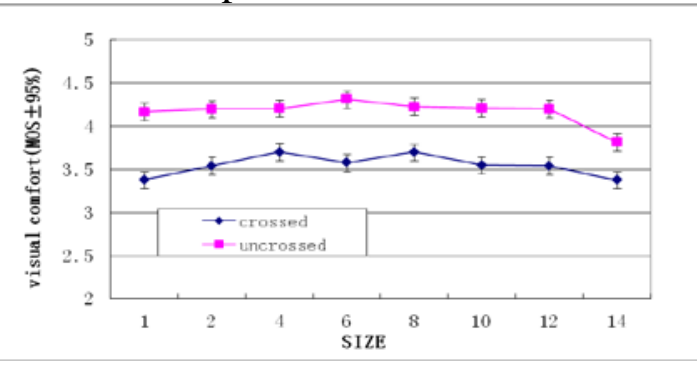

( b )

Fig. 3 Results of the size experiment about size on visual comfort

At different disparity magnitude and parallax type, the fitting lines were obtained by analysing the relationship between normalized mean opinion score (NMOS) of visual comfort and normalized size (NS), The R2 of decisive factor, is the fit index of the fitting line and sample observation value. The Function of the fitting lines are shown in table 2. 
Table 2 The Function of the fitting lines of NMOS and NS

\begin{tabular}{|l|l|l|}
\hline Disparity condition & Function of the fitting line & $\mathrm{R}^{2}$ \\
\hline Dis_3\% crossed & NMOS $=-0.0089 N S 3+0.0708 N S 2-0.0264 N S+0.2353$ & 0.7156 \\
\hline Dis_3\% uncrossed & NMOS $=-0.0171 N S 3+0.2145 N S 2-0.8276 N S+1.6207$ & 0.8435 \\
\hline Dis_5\% crossed & NMOS $=0.0132 N S 3-0.2213 N S 2+1.0101 N S-0.5495$ & 0.6873 \\
\hline Dis_5\% uncrossed & NMOS $=-0.0095 N S 3+0.0554 N S 2+0.1719 N S+0.0128$ & 0.9182 \\
\hline Dis_7\% crossed & NMOS $=0.0117 N S 3-0.1983 N S 2+0.9931 N S-0.7937$ & 0.6271 \\
\hline Dis_7\% uncrossed & NMOS $=0.00009 N S 3-0.0308 N S 2+0.1813 N S+0.5567$ & 0.6807 \\
\hline
\end{tabular}

The effect of disparity distribution on visual comfort of stereoscopic images. There were 17 observers' rating scores available for this experiment, Through a repeatable double factor analysis of variance, for the range of disparity distribution of the two main part, $\mathrm{F}(4,64)=19.604, \mathrm{P}<0.01$, which shows the factor of disparity range is important. For the disparity space distribution type, $\mathrm{F}$ $(4,64)=8.397, \mathrm{P}<0.01$, means it is also a significant factor. Fig. 4 shows the main experimental results of MOS, and the influence trends of the two factors in different disparity conditions. Overall, the rating scores would reduce when there is crossed disparity. The larger the disparity distribution range is, the faster it dropped. When the disparity range reach or exceed 4\% (40 arc min), the five distribution types will all cause visual discomfort. In our experiment, type_c is a special distribution, the visual comfort degree is always highest at the same disparity distribution range, and this situation may be caused by the reason that the magnitude of the crossed and uncrossed disparity is lower than other types at this time.

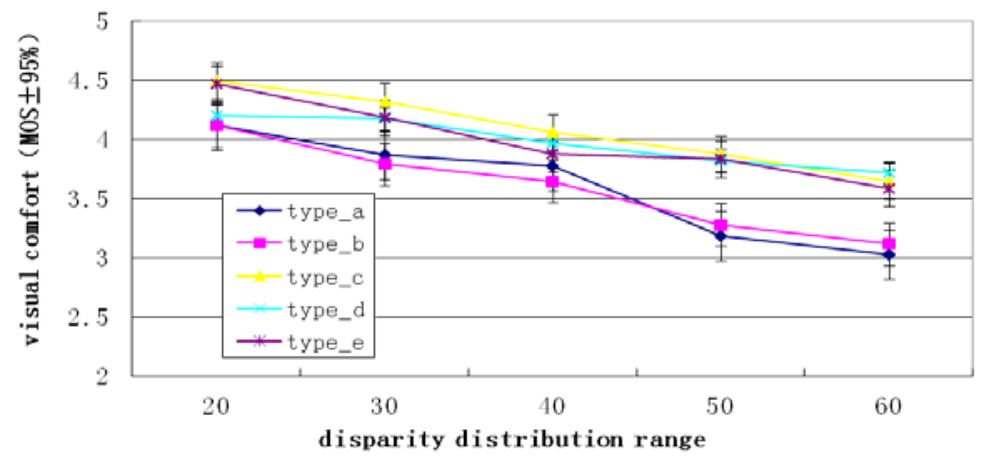

Fig.4 Results of the disparity distribution experiment about size on visual comfort

Based on the different disparity distribution types, the relationship between normalized mean opinion score (NMOS) of visual comfort and normalized disparity distribution (NDIS) was settled by fitting line, as is shown in Table 3.

Table 3 The function of the fitting lines of NMOS and NDIS

\begin{tabular}{|c|c|c|}
\hline Disparity distribution type & Function of the fitting line & $\mathrm{R}^{2}$ \\
\hline Type a & NMOS $=-0.2624$ NDIS +1.3083 & 0.9399 \\
\hline Type $b$ & NMOS $=-0.2513$ NDIS +1.2278 & 0.9839 \\
\hline Type c & NMOS $=-0.2526$ NDIS +1.2664 & 0.9969 \\
\hline Type d & NMOS $=-0.2725$ NDIS +1.3517 & 0.9655 \\
\hline Type e & NMOS $=-0.2401$ NDIS +1.1785 & 0.9604 \\
\hline
\end{tabular}

\section{Conclusion}

This paper mainly investigated the effect of the main part size and disparity distribution type of stereoscopic images on visual comfort through two subjective assessment experiments. The experimental results show that the main part size and disparity distribution type are important factors which can affect the visual comfort. In the first experiment, when the size of the main part is too large or too small, it would always make the observer feel uncomfortable. In addition, the body 
size in different disparity conditions is also different, when the disparity magnitude is too large, to get the more comfort viewing experience, the size should be smaller. The experimental study of the disparity distribution type has proved that when there is a crossed disparity element in the image, the comfort degree decreased, and the larger the disparity distribution range is, the faster it dropped. The observers would feel more comfortable when the disparity range is small. In addition, in most cases, the visual comfort degree is the highest when there is a crossed and uncrossed disparity element in the stereoscopic image.

Our experiments have important guiding significance for the establishment and improvement of the relevant objective evaluation experimental model and acquisition and display technology of stereoscopic images.

\section{Acknowledgement}

This research was financially supported by the national science and technology planning project "Study and application demonstration on the key technology of the stage effect"(Item Number: 2012BAH38F00).

\section{References}

[1]Ren Hui, Su Zhibin,Lv Chaohui, Zou Fangju. Effect of Region Contrast on Visual Comfort of Stereoscopic Images[J]. Electronic letteres, 2015,51 (13) 983-985.

[2]Robert S. Kennedy, Norman E. Lane, Kevin S. Berbaum \& Michael G. Lilienthal. Simulator sickness questionnaire: an enhanced method for quantifying simulator sickness[J]. The International Journal of Aviation Psychology,1993, 3 (03) 203-220.

[3] Wa James Tam, Filippo Speranza, Sumio Yano, Koichi Shimono, and Hiroshi Ono. Stereoscopic 3D-TV: Visual Comfort[J]. Broadcasting,IEEE Transactions on.,2011,57 (2) 335-346.

[4]Donghyun Kim, Kwanghoon Sohn. Visual Fatigue Prediction for Stereoscopic Image[J]. Circuits and Systems for Video Technology, IEEE Transactions on.,2011,21 (02) 231-236.

[5]Hosik Sohn, Yong Ju Jung ; Seong-il Lee ; HyunWook Park. Attention model-based visual comfort assessment for stereoscopic depth perception[J].Digital Signal Processing (DSP),17th International Conference on,2011. 1-6.

[6]Wang Qin, Wang Qionghua., Liu Chunling. Effects of parallax and spatial frequency on visual comfort in autostereoscopic display'. Journal of Optoelectronics·Laser.,2012, 23 (08) 1604-1608.

[7]Y. Nojiri, H. Yamanoue, S. Ide, S. Yano, and F. Okano. Parallax distribution and visual comfort on stereoscopic HDTV[J]. In Proceedings of IBC, 2006, 373-380.

[8]Chang B. The study on objective evaluation algorithm of stereo video comfort. Xi`an University of Science and Technology. 2013.

[9]GB/T 7410-1987, Method of subjective assessment of quality of colour TV pictures, 1987. 